\title{
Correlation between interphase and metaphase chromosome arrangements as studied by laser-uv-microbeam experiments*
}

\author{
T. Cremer, H. Baumann, K. Nakanishit and \\ C. Cremer
}

Institute of Anthropology and Human Genetics, Unlversity of Heidelberg, D-6900 Heidelberg, FRG, 'Department of Pathology, Kyoto Prefecturel University of Medicine, Kyoto, Japan and Institute of Applied Physics I, University of Heidelberg, D-6900 Heidelberg, FRG

In spite of many attempts at resolution, the problem of the chromosome topography in interphase nuclel and 1 ts possible functional signiflcance (Lewin, 1981, Vogel and Krluger, 1983) has remained a matter of great controversy and uncertainty (for reviews see comings,1980, Avivi Feldman, 1980). In particular, the relationship between metaphase and interphase arrangements of chromosomes is still largely unknown. A solution of this problem seems especially urgent, since the spatial relationship of chromosones at metaphase has been extensively studied in many species during the last twenty years. Recently, Bennett and coworkers have demonstrated a highly ordered arrangement of centromeres in metaphase plates of certain plant species (Bennett, 1983) and atimulated fresh interest in the development of techniques for mapping the arrangements of individual chromosomes directly within the interphase nucleus. In this paper we describe a laser-uv-microbeam approach which allows selective microirradiation of a small amount of chromatin in the interphase nucleus of cultured cells and study of its distribution in chromosomes during the subsequent mitosis. Alternatively, chromatin can be microircadiated ouring mitosis and its distribution can be studied in the subsequent interphase nucleus.

* supported by the Deutsche Forschungsgemeinschaft, Bonn. Dr. R. Nakanishi was on a Fellowship of the A. von Humboldt stiftung.

Chromosomes todoy, volume 8 George Allen \& Unwin 1984 
TERRITORIAL ORGANIZATION OF CHROMOSOMBS IN THE INTERPEASE NUCLEUS OF CULTURED CHINESB HAMSTER CBLLS

A prerequisite to any meaningful conclusion which might be drawn from atudies of chromosome arrangements at metaphase, on the arrangement of chromosomes in the interphase nucleus, is the assumption that each chromosome continues to exist in the interphase nucleus as a spatially distinct, and in spite of its decondensation, still relatively compact entity. In this case, each single chromosome would occupy a small and coherent domain of the total nuclear space. The hypothesis that such chromosome territories (also called chromosome domains) exist in the interphase nucleus has been put forward already by Rabl (1885) and Boveri (1909). Other models, however, proposed half a century later, have still seriously considered the possibility that the chromatin fibres of euchromatic regions within individual chromosomes might become extensively diepersed in the interphase nucleus (Comings, 1968, vogel and schroeder, 1974). In case that the chromatin fibre, which is supposed to constitute each individual chromosome, would be extended throughout a major part of the total nuclear space, each chromosome would form associations with many other chromosomes at many sites. If so, chromosome arrangements at metaphase would naturally yield an insufficient picture of their interphase arrangements.

Laser-uv-microirradiation $(\lambda=257 \mathrm{~nm})$ has provided the possibillty to put such models to a rigorous test. Small parts of interphase nuclei of cultured Chinese hamster cells were microlrcadiated. Obviously, the distribution of microirradiated chromatin in the chromosomes of the subsequent metaphase critically depends on the actual arrangement of interphase chromosomes in the microirradiated nuclear part. In the case of a territorial organization, it 1s predicted that microirradiated chromatin is restricted to a few metaphase chromosomes, depending on the size of the microirradiated nuclear segment. In contrast, extensive dispersion of the chromatin fibres from individual chromosomes should be indicated by scattering of microirradiated chromatin acrose a correspondingly increasing number of metaphase chromosomes.

Microirradiated chromatin was Identified either autoradiogra- 
phically by detection of unscheduled DNA synthesis (UDS) which takes place during the course of excision repair of DNA photolesions in the microirradiated nuclear segment (zorn et al., 1979; Cremer et al., 1982a) or by use of antibodles specific for the detection mainly of pyrimidine dimers in a DNA structure which
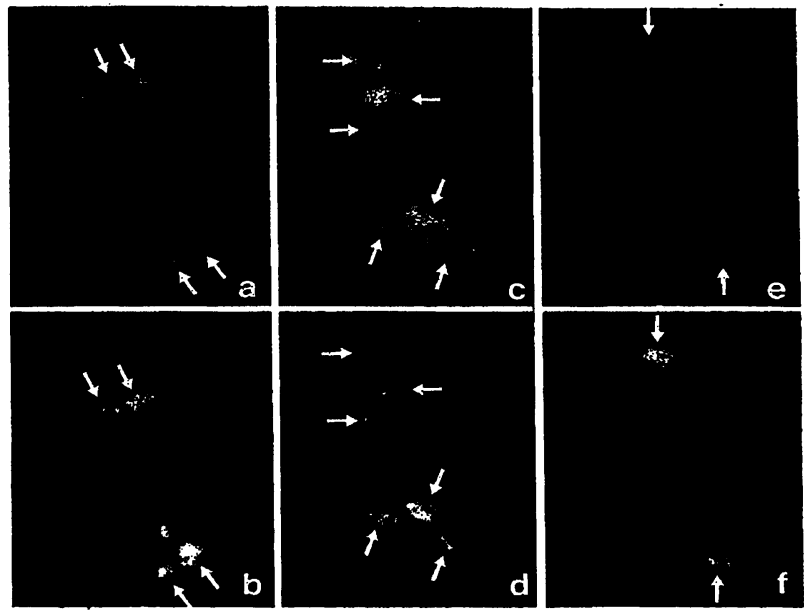

Fig. 1: Pairs of daughter nuclei were fixed in acetic acid/methanol $(1: 3) 3 h 5^{\prime} a, 3 h 25^{\prime} c$ and $4 \mathrm{~h} 25^{\prime}$ e after microirradiation of a small part of the metaphase plate of Chinese hamster cells (M3-1) . a,c,e Nuclei stained with DAPI. b,d,f Immunocytochemical localization of microlrradiated chromatin (arrows). Staining for Indirect immunofluorescence microscopy was performed as previously described (Cremer et al., 1983). Antibodies with a high affinity for uv-irradiated DNA were raised independantly in rabbits by Dr. K. Nakanishi and Dr. J.J. Cornelis. Both antisera yielded identical results. Goat-anti-rabbit IgG conjugated with fluoresceine isothiocyanate (FITC) was obtained from Nordic. For comparison nuclel were counterstalned with DAPI, thereafter. By approprlate filtering, the blue DAPI fluorescence could easily be distinguished from the green immunofluorescence. Microphotopraphs were taken with a zeiss photomicroscope equipped with eplfluorescence illumination. A mirrorlike distribution of microirradlated chromatin in each pair of daughter nuclel becomes more obvious when changes in the positions of the whole nuclei during the postincubation period are taken into consideration and nuclei are accordingly repositioned. 
represent the major DNA-photoproduct of uv-irradiation at $257 \mathrm{~nm}$ (Cremer et al., 1983, Bens et al., 1983). With both methods of detection we found that microirraditation of a small part of the interphase nucleus (approx. 5 of the total nuclear area) in different lines of chinese hanster cells, resulted in intensive labelling of segments from a few chromosomes only ( 3 to 4 on the average), while the majority of chromosomes in each metaphase spread (modal numbers 22 - 23) remained unlabelled. It is important to note that this result was obtained both after microirradiation of nuclel in G1 (Cremer et al., 1982a) and in s-phase (Hens et al.. 1983), indicating that a territorial organization of chromosomes is maintained during subsequent stages of the cell cycle. Furthermore, It was found that the number of chromosomes in which formation of sister chromatid exchanges (BCEs) was induced at a given uv-incident energy Increased significantly with the size of the microirradiated nuclear area (Raith et al., 1984). In addition, mitotic chromosomes were microirradiated either at metaphase (see below) or at anaphase (Hens et al., 1983). In the subsequently formed interphase nuclei the microlrradiated chromatin was limited to a small volume (FIg. 1). In conclusion these microbearn experiments support the concept that interphase chromosomes occupy distinct domains of the interphase nucleus.

MIRROR-LIKB DISTRIBUTION OF CHROMATIN IN PAIRS OF DAUGTHER NOCLEI

Microirraditaion of metaphase chromosomes in living Chinese hamster cells allowed us to test a hypothesis which was proposed for the first time by Theodor Boveri (1888, 1909). Bover 1 stated that arrangements of chromosomes in pairs of daughter nuclei would occur in a mirror-like fashion. This hypothesis was based on observations made during the first cell cycles of fertilized eggs of Ascaris megalocephala. Boveri found that the arrangements of prophase chromosomes were mirror like in each pair of daughter cells derived from the same mitotic event, while they appeared variable to a large extent in cells from different mitotic events. By means of immunofluorescence microscopy we have visualized the distribution of microirradiated chromatin in a sample of 27 pairs of daugh- 
ter nuclei obtained after microirradition of a small part of the metaphase plate (see Fig. 1 for examples). To test the similarity of the chromatin distribution in each pair, the daughter nuclei were projected over each other in a mirror like fashion and positio-
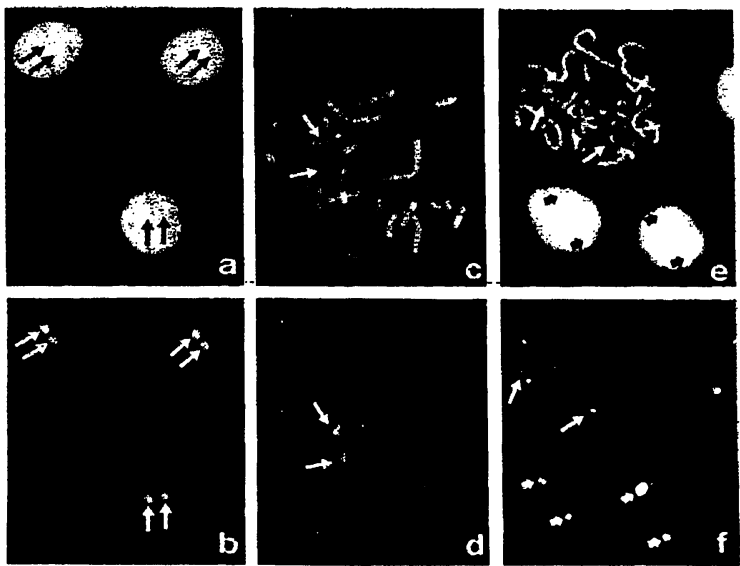

Fig. 2s Microirradiation of interphase nuclei at two sites of Chinese hamster cells (M3-1) was performed six hours after release of the cells from a thymidine block (2 mM). Chromosome preparations were performed in situ as previously described (zorn et al., 1979) after a postincubation period of 4 hours in the presence of caffeine $(2 \mathrm{mM})$. a,c,e Interphase nuclei and metaphase spreads stained with DAPI. b,d,e Imnunocytochemical localization of microirradiated chromatin. (For details see legend to Fig. 1) Arrows indicate corresponding sites of microirradiated chromatin in a,b, c,d and e,f. a,b Microirradiation at two nuclear sites close to each other. C,d Metaphase spread obtained after microirradiation of the nucleus in the preceding interphase at two sites close to each other. e,f Metaphase spread obtained after microirradiation of the interphase nucleus at two sites far apart from each other as demonstrated by two adjacent nuclei. Notably, two sites of indirect immunofluorescence corresponding with two sites of partial chromosome shattering (PCS) (Cremer et al., 1983) can be detected in the two metaphase spreads. In contrast, after microirradiation of the interphase nucleus at one site indirect immunofluorescence corresponding to one site of PCS was generally observed in the subsequent metaphase (Hens et al., 1983). 
ned In a way that yielded an overlap of both the total nuclear areas and the immunoflourescent areas as complete as possible. For comparison, 27 pairs of non-daughter nuclei of similar size were tested from the same sample. In support of Boveri's hypothesis, we found that the extent to which the Immunoeluorescent areas overlapped was significantly larger (by a factor of approx. 2) in pairs of daughter nuclei. (R. Nakanish1 T. Cremer, in preparation).
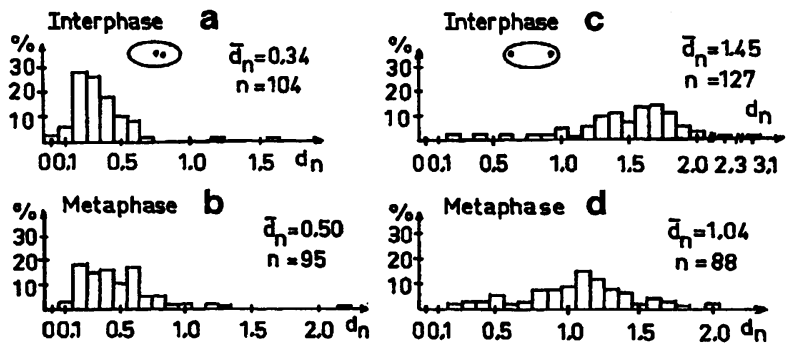

Fig. 38 Comparison of relative distances $d_{n}$ in interphase $a_{, c}$ and metaphase $b$,d between the two sites of microirradiated chromatin in Chinese hamster cells (M3-1) (for further detalls compare Fig. 2) . In each interphase nucleus and metaphase spread the distance d between the centers of the two immunofluorescent sites corresponding to the two sites of microlrradiated chromatin was determined. Nuclel and metaphase spreads were considered as ellipses and the diameters of the two major axes $a$ and $b$ were also measured. To avold any prefudice, evaluation of metaphase spreads was performed by a person without information on the particular type of microirradiation. To account for variations in size of nuclei and metaphase spreads d was divided by $1 / 2 \sqrt{\mathrm{ab}}$ in each case. $1 / 2 \sqrt{\mathrm{ab}}$ corresponds to the radius of a circle of the same area. al Frequency distribution of $d_{n}$ in a sample of 104 interphase nuclei with two sites of microIrradiation close to each other. Nuclei were situated close to the metaphase spreads evaluated in b (compare Fig. $2 a, b$ ). b) Frequency distribution of $d_{n}$ in 95 metaphase spreads obtained 4-6 hours after microirradiation of nuclel in 4419 cells at two sites as described in a (compare Eig. $2 \mathrm{c}, \mathrm{d}$ ). d) Frequency distribution of $d_{n}$ in a sanple of 127 interphase nuclel situated close to metaphase spreads evaluated in d and microirradiated at two sites far apart from each other. d) Frequency distribution of $d_{n}$ in 88 metaphase spreads obtained 4-6 hours after microlrradiation of the interphase nucleus In 4350 cells as described in $c$ (compare Fig. $2 \mathrm{e}, \boldsymbol{F}$ ). Note that the mean values of $d_{n}$ in metaphase spreads still reflect the distances between the two sites of microlrradiation in the interphase nucleus. The difference between $d_{n}$ in $a, c$ (interphase nuclei) and $b_{,} a$ (metaphase opreads) is highly signifioant ( $P<0.001$ ) 
STATIC ARRANGEMENT OF CHROMOSOME TERRITORIES IN INTERPHASE NUCLEI OF CULTURED CHINESE HAMSTER AND HUMAN FIBROBLASTOID CELLS

Theodor Boveri (1909) was also the first to predict that the chromosome territories would be rather rigidiy fixed during interphase. Notably, cell cycle transit times in the developing egg of Ascaris megalocephala from which Boveri's hypothesis was derived are rather short and many decades later the possibility of extensive alterations in chromosome order during subsequent stages of the cell cycle was still taken into consideration as one possibility among others for other cell types (Vogel and schroeder, 1974). In case of a dynamic interphase chromosome arrangement, studies of metaphase chromosome arrangements could at best provide information on interphase chromosome arrangements at a late stage of the cell cycle. We, therefore, decided to relnvestigate this problem both in fibroblastoid Chinese hamster cells (CHL) (Cremer et al., 1982a,b) and in human fibroblastoid cells (Flow 2000, population doubling level approx. 30). Interphase nuclel were microirradiated at two sites and cells were either fixed immediately thereafter or allowed to grow for an additional postincubation period (20 and 40 hours in case of CHL-cells and 10 and 20 hours in case of human cells). Microirradiated chromatin was made visible as described above either by uDs-labelling or by immunofluorescent labelling. Our data are consistent with Boveri's early hypothesis. Distances between the two sites of microirradiated chromatin changed only slightly in CHL-cells (Cremex et al., 1982 a and appeared practically constant in human cells (K. Nakanishi \&. Cremer, in preparation).

CORRELATION BETWEEN CHROMOSOME POSITIONS IN INTERPHASE AND METAPRASE

The arrangement of chromosome territories in the interphase nucleus is expected to differ from their order in metaphase preparations. Factors which disrupt the interphase order include condensation of chromosomes during prophase and chromosome movements involved in the metaphase plate formation. In addition, techniques routinely used to obtain metaphase spreads suitable for karyotyping (including spindle polsons, hypotonic treatment and spreading of 


\section{Study of chromosome arrangements}

chromosomes) may destroy the chromosome arrangement in the intact metaphase plate to an extent which is difficult to control. It should also be noted that chromosomes in a metaphase spread are arranged in a plane, while their respective territorles were arranged in the threedimensional structure of the preceding interphase nucleus. In spite of these limitations a statistical evaluation of distances between metaphase chromosomes has been attemped by many authors, and conflicting results have been published. Therefore, we found it important to Investigate whether such distances would reflect, if only to a limited extent, the distances between the respective chromosome territories in the preceding interphase. If such a correlation could indeed be demonstrated even for metaphase spreads obtained by routine preparation procedures, the case of the metaphase plate as a substitute for the investigation of interphase chromosome arrangements could be strenghtened, especially under conditions which do not disrupt its natural arrangement by crude opreading techniques.

ont1l recently, the possiblity to study such a correlation was restricted to a few cases in which heterochromatic regions of particular chromosomes could be studied in mitosis as well as in interphase (e.g. Pera \& Schwarzacher, 1970). As a general test of this correlation we have microirradiated two sites in interphase nuclel of Chinese hamster cells (MB-1); either close to or far apart from each other. Microirradiated chromatin served as a probe to study its respective position in metaphase preparations by indirect Immunofluorescence microscopy (Figs. 2 and 3; see legends for further experimental details). Both interphase nuclei and metaphase preparations consistantly revealed two distinct siter of immunofluorescent staining (Fig. 2). Distances between these two sites were measured in enlarged photographic prints and corrected with regard to the varying diameters of nuclei and metaphase spreads (see legend to Fig. 3). The results of these measurements show that the mean distances between the two sites of microirradiated chromatin in the interphase nucleus are reflected by their corresponding distances in the subsequent metaphase spread. Another series of experiments was performed in essentially the same way except that metaphase plates were fixed with acetic acid/methanol 
$(1: 3)$ without colchicine pretreatment and hypotonic shock. Preliminary data of this series further support the concept that chromosome order in the undisturbed metaphase plate might indeed refleot the relative positions of chromosome territories in the preceding interphase nucleus to a considerable extent. A full account of these experiments will be published elsewhere (H. Baumann, T. Cremer, N. Bulbuc \& J.J. Cornelis, in preparation).

F1g. 2 c-f clearly shows shattering of metaphase chromosomes corresponding with the sites of immunofluorescent staining. We have termed this effect as partial chromosome shattering (PCs) (Cremer et al., 1983) and auggest that it might at least partly be due to an undercondensation of microirradiated chromosome segments. This In turn could explain why several chromosomes microirradiated at one site of the interphase nucleus were often closely associated at one site of PCS in the subsequent metaphase (Hens et al., 1983). shattering resulted from the synerglotic effect of caffeine (2mM) on chromosome damage in microirradiated cells (Cremer et al.. 1983). When caffeine was omitted from the postincubation medium chromosomal damage was much less pronounced. However, immunofluorescent labelling in these metaphase spreads generally was too weak for an unequivocal identification of the microirradiated chromatin. Possibly, antigenic sites become masked when microirradiated chromosomes condense normally in the absence of caffeine.

\section{MAPPING OF INTERPHASE POSITIONS OF INDIVIDUAL CHROMOSOMES}

In a recently published series of experiments (Cremer et al., 1982 b), the interphase nucleus of CHL-cells was microirradiated at one site either at 1ts central part or at its perhiphery. Metaphase spreads with PCs obtained after postincubation with caffeine 10.5 $\mathrm{mix}$ ) were karyotyped. No significant difference was observed between the frequencles of damage obtained for individual chromosomes at elther site of microirradiation. Interestingly, while an average of 5 - 6 damaged chromosomes per metaphase was found in these experiments, the frequency of joint damage of homologous chromosomes was low, arguing against somatic pairing of homologuos chromosomes as a frequent event in this cell type. In spite of their limitation, 
these data demonstrate the feasibility of the microbeam approach for mapping the posititons of individual chromosomes in the interphase nucleus.

Avivi, L. and Feldman, M. 1980. Arrangement of chromosomes in the interphase nucleus of plants. Hum. Genet. 55, 281-295.

Bennett. K.D. 1983. The spatial distribution of chromosomes. In Kew Chromosome conference II, P.E. Brandham and M.D. Bennett eds., 71-79, London: George Allen \& Unwin.

Bover $i$, Th. 1888, Zelien studien. Jena. $z$. Naturw. 22, 687-882.

Bover1. Th. 1909. Die Blastomerenkerne von Ascaris megalocephala und die Theorie der Chromosomenindividualităt. Arch. zellfor sch. 3, 181-268.

Comings, D.E. 1968. The rationale for an ordererd arrangement of chromatin in the interphase nucleus. Am. J. Hum. Genet. 20, 440-460.

Comings, D.F. 1980. Arrangement of chromatin in the interphase nucleus. Hum. Genet. $53,131-143$.

Cremer, T., Cremer, C., Baumann, H., Luedtke, E.-K., Sperling, R., Teuber, V. and zorn, C. 1982 a. Rabl's model of the interphase chromosome arrangement tested in Chinese hamster cells by premature chronosome condensation and laser-uv-microbeam experiments. Hum. Genet. 60, 46-56.

Cremer, T., Cremer, C., Schnelder, T., Baumann, H., Bens, L. and Kirsch-Volders, M. 1982b. Analysis of chromosome positions in the interphase nucleus of Chinese hamster cells by laser-uvmicroirradiation experiments. Hum. Genet. 62, 201-209.

Cremer, C., Cremer, T., Bens, L., Baumann, H., Cornelis, J.J. and Nakanishi, K. 1983. OV microirradiation of the Chinese hamster cell nucleus and caffeine post-treatment. Immunocytochemical localization of DNA photolesions in cells with partial and generalized chromosome shattering. Mutat. Res, 107, 465-476.

Hens, L., Baumann, E., Cremer, T., sutter, A., Cornelis, J.J. and cremer, C. 1983. Immunocytochemical localization of chromatin regions uv-microirradiated in s-phase or anaphase. Bvidence for a territorial organization of chromosomes during cell cycle of cultured Chinese hanster cells. Exp. Cell Res., in press.

Lewin, R. 1981. Do chromosomes cross talk? Sclence 214, 1334-1335.

Pera, F. and schwarzacher, H.G. 1970. Localization of the heterochromatic chromosomes of Mlcrotus agrestis in interphase and mitosis. Cytobiology 2, 188-199.

Rabl, C. 1885. Über zelltheilung. Morph. Jb. 10, 214-330.

Raith, M., Cremer, T., Cremer, C. and speit, G. 1984. Sister chromatid exchange (SCE) induced by laser-uv-microlrradiation. Correlation between the distribution of photolesions and the distribution of sCEs. Submitted for publication.

vogel, F. and schroeder, T.M. 1974. The internal order of the interphase nucleus. Hum. Genet. 25, 265-297.

Vogel, F. and Krliger, J. 1983. Is there a general relationship between estimated chromosome diatances in interphase and location of genes with related functions? Aum. Genet. $63,362-368$.

zorn, C., Cremer, C., Cremer, T. and zimuer, J. 1969. Unscheduled DNA synthesis after partial uv-irradiation of the cell nucleus. Distribution in interphase and metaphase. Bxp. Cell Res, 124, 111-119. 